CERN-TH.7069/93

PRA-HEP 93/15

hep-th/9311012

\title{
Duality invariant class of exact string backgrounds
}

\author{
C. Klimčík* \\ Theory Division, Nuclear Centre, Charles University, \\ 18000 Prague 8, Czech Republic \\ and \\ A.A. Tseytlin** \\ Theory Division, CERN \\ CH-1211 Geneva 23, Switzerland \\ and \\ Theory Group, Blackett Laboratory, Imperial College \\ London SW7 2BZ, U.K.
}

We consider a class of $2+D$ - dimensional string backgrounds with a target space metric having a covariantly constant null Killing vector and flat 'transverse' part. The corresponding sigma models are invariant under $D$ abelian isometries and are transformed by $O(D, D)$ duality into models belonging to the same class. The leading-order solutions of the conformal invariance equations (metric, antisymmetric tensor and dilaton), as well as the action of $O(D, D)$ duality transformations on them, are exact, i.e. are not modified by $\alpha^{\prime}$-corrections. This makes a discussion of different space-time representations of the same string solution (related by $O(D, D \mid Z)$ duality subgroup) rather explicit. We show that the $O(D, D)$ duality may connect curved $2+D$-dimensional backgrounds with solutions having flat metric but, in general, non-trivial antisymmetric tensor and dilaton. We discuss several particular examples including the $2+D=4$ - dimensional background that was recently interpreted in terms of a WZW model.

CERN-TH.7069/93

November 1993

* e-mail: presov@cspuni12.bitnet

** On leave from Lebedev Physics Institute, Moscow, Russia. e-mail: tseytlin@surya3.cern.ch and tseytlin@ic.ac.uk 
1. The aim of the present paper is to study the action of the duality transformations on a simple class of exact string solutions which have a covariantly constant null Killing vector. Such ('plane wave' type) solutions of Einstein equations are well known [1]. Some particular examples of such spaces were found to be solutions of the string effective equations to all orders of $\alpha^{\prime}$ perturbation theory [2] [3] [4] [5] [6] [6] [8]. In the case when the 'transverse' space is not described by a conformal theory (e.g. is not flat) one finds more general solutions with the 'transverse' couplings satisfying the first order renormalisation group - type equations (with the light-cone coordinate playing the role of the RG time) [9] [10]. The advantage of the 'plane wave' backgrounds is their simplicity and controllable (or vanishing) quantum $\alpha^{\prime}$-corrections. This suggests that duality transformations acting on such backgrounds should also take an explicit form. 1

As it is well known, string solutions with $D$ abelian isometries are transformed into

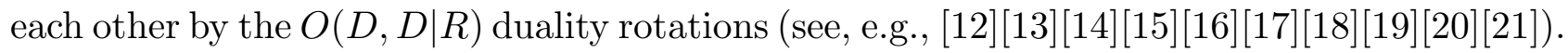
The origin of this symmetry is in the $2 d$ duality on the string world sheet that relates 'left' and 'right' parts of the isometric string coordinates leading to the $O(D) \times O(D)$ symmetry in the correlators of vertex operators and thus (extended off shell) in the string field theory [18] or string effective action. Combining the $O(D) \times O(D)$ rotations with the obvious $G L(D, R)$ coordinate transformations and constant shifts of the antisymmetric tensor one finds the $O(D, D \mid R)$ duality group. This symmetry becomes manifest in the phase-space approach [12] [17] or closely related (non-manifestly Lorentz invariant) Lagrangian approach [22]. From the sigma model path integral point of view, one is able to make the formal $2 d$ duality transformation (via introducing Lagrange multipliers or gauging) that transforms one sigma model into its $O(D, D \mid R)$ rotation [14] [19] [20]. Under special conditions (compactness of orbits of isometries) the transformations from the $O(D, D \mid Z)$ subgroup relate the backgrounds that correspond to the same conformal field theory [19][20].

1 Simple one-isometry duality transformations of the 'plane wave' backgrounds were previously discussed in [11]. 
In general, the leading-order duality preserves only the one-loop conformal invariance equations, i.e. transforms the leading-order string solutions into the leading-order ones (or exact solutions into leading-order ones). In fact, the leading-order duality transformation rules are known to be modified by $\alpha^{\prime}$-corrections [15] [23]. However, such $\alpha^{\prime}$-corrections may be absent for certain backgrounds. Then the leading-order duality transformations would relate exact string vacua. A recent example is provided by the duality in the $S L(2, R) / R$ $2 d$ 'black hole' background [24][15] which remains semiclassical in a specific scheme [25]. Similar result is true for the duality in the $[S L(2, R) \times R] / R$ gauged WZW model $(3 d$ 'black string' [26]) which also retains its semiclassical form in a special scheme [27] [28].

2. Below we shall present a simpler and more explicit example of a situation when the leading-order duality transformations are actually exact. Consider a sigma model with coordinates $x^{i}$ describing a flat Euclidean signature (or toroidal [29]) background parametrised by constants $g_{i j}, b_{i j}, \phi(i, j=1, \ldots, D)$. Coupling it to an additional sigma model with coordinates $t^{a}$ (i.e. making $g, b, \phi$ dependent on $t^{a}$ ) one gets a class of models with $D$ abelian isometries which is invariant under $O(D, D \mid R)$ duality. possibility is to consider a class of cosmological backgrounds by taking $t^{a}$ to be just one time coordinate. The resulting leading-order term in the string effective action and the corresponding field equations for $g(t), b(t), \phi(t)$ are explicitly invariant under the duality [17. However, both the solutions and their duality rotations in this case are modified by $\alpha^{\prime}$-corrections and their exact form is not known explicitly.

Instead, we shall take $t^{a}$ to be the pair of light-cone coordinates $(u, v)$ and impose the null Killing symmetry by letting the background fields to depend only on $u$, i.e.

$$
I(u, v, x)=\frac{1}{\pi \alpha^{\prime}} \int d^{2} z\left\{-2 \partial u \bar{\partial} v+\left(g_{i j}+b_{i j}\right)(u) \partial x^{i} \bar{\partial} x^{j}\right\}+\frac{1}{4 \pi} \int d^{2} z \sqrt{\gamma} R^{(2)} \phi(u) .
$$

2 In general, one can also introduce 'non-diagonal' terms like $A_{a i} \partial t^{a} \bar{\partial} x^{i}$ (see e.g. 20]) which we shall ignore. 
The only non-zero components of the connection, curvature and $H_{\mu \nu \kappa}=\left(\partial_{\mu} b_{\nu \kappa}+\right.$ cycle) $\left(x^{\mu}=u, v, x^{i}\right)$ are

$$
\begin{gathered}
\Gamma_{i j}^{v}=\frac{1}{2} \dot{g}_{i j}, \quad \Gamma_{j u}^{i}=\frac{1}{2} g^{i k} \dot{g}_{k j}, \quad \dot{F} \equiv \frac{d F}{d u}, \\
R_{i u j u}=-\frac{1}{2} \ddot{g}_{i j}+\frac{1}{4} g^{m n} \dot{g}_{i m} \dot{g}_{n j}, \quad H_{u i j}=\dot{b}_{i j},
\end{gathered}
$$

so it is easy to show that the one-loop conformal invariance equations

$$
\bar{\beta}_{\mu \nu}^{G}=R_{\mu \nu}-\frac{1}{4} H_{\mu \kappa \lambda} H_{\nu}{ }^{\kappa \lambda}+2 D_{\mu} D_{\nu} \phi=0, \quad \bar{\beta}_{\mu \nu}^{B}=-\frac{1}{2} D^{\lambda} H_{\lambda \mu \nu}+\partial^{\lambda} \phi H_{\lambda \mu \nu}=0,
$$

reduce just to one equation $\left(\bar{\beta}_{u u}^{G}=0\right)^{3}$

$$
-\frac{1}{2} g^{i j} \ddot{g}_{i j}+\frac{1}{4} g^{i j} g^{m n} \dot{g}_{i m} \dot{g}_{j n}-\frac{1}{4} g^{i j} g^{m n} \dot{b}_{i m} \dot{b}_{j n}+2 \ddot{\phi}=0
$$

Moreover, there are no $\alpha^{\prime}$-corrections to the $\bar{\beta}^{G}=\bar{\beta}^{B}=0$ equations on this background: all higher-order contractions of relevant tensors vanish [5] [10]. This is also obvious from the path integral point of view [3]: the field $v$ in (1) plays the role of a Lagrange multiplier that 'freezes' out fluctuations of $u$ unless there are sources corresponding to the $u$-direction. Once the one-loop condition of conformal invariance in the $u$-direction (3) is satisfied, it is easy to argue from the structure of (1) that no higher-loop divergences can appear. Since all the scalar invariants vanish, there are no contributions to the central charge, i.e. $c=2+D$ 目

3 The most general $D+2$ dimensional Minkowski signature metric admitting a covariantly constant null Killing vector can be represented as $d s^{2}=G_{\mu \nu} d x^{\mu} d x^{\nu}=-2 d u d v+g_{i j}(u, x) d x^{i} d x^{j}$. When the transverse metric is flat one can make a coordinate transformation to put the full metric into the form: $d s^{2}=-2 d u d v+d x^{i} d x_{i}+2 A_{i}(u, x) d x^{i} d u+K(u, x) d u^{2}$. As explained in [10] exact solutions of the string equations with $g_{i j}=\delta_{i j}, A_{i}=-\frac{1}{2} F_{i j}(u) x^{j}, K=k_{i j} x^{i} x^{j}+k_{0}$, considered in [3] [4] are equivalent to the solutions with $g_{i j}=g_{i j}(u)$ in [6] [4]. Equivalent representations for $B_{\mu \nu}$ are $B_{i u}=-\frac{1}{2} \dot{b}_{i j} x^{j}, B_{i j}=0$ and $B_{i u}=0, B_{i j}=b_{i j}(u)$. Note that in our model (1) $B_{\mu v}=0$ since if $B_{\mu v}=B_{\mu v}(u) \neq 0$ there are non-vanishing higher order $\alpha^{\prime}$-corrections to the string field equations.

4 In contrast to the case of non-conformal 'transverse' theory [9] [10] here one cannot include the linear $p v$-term in $\phi$ since this leads to the conditions $p \dot{g}_{i j}=0, p \dot{b}_{i j}=0$. 
The solutions of (3) thus represent exact string backgrounds. Since we have just one equation for $D^{2}+1$ unknown functions $g_{i j}(u), b_{i j}(u), \phi(u)$ there are many particular solutions. For example, we can set $b_{i j}=$ const or $\phi=$ const or $g_{i j}=\delta_{i j}$. The latter case looks the simplest since it corresponds to the flat $2+D$ dimensional space (and in this sense is analogous to the linear dilaton background [30]) with the conformal invariance (3) being maintained by the balance of contributions of non-constant antisymmetric tensor and dilaton fields. In contrast to the case of the linear dilaton vacuum here the propagation of the classical string is non-trivial being effected by the $b_{i j}$ background. As for the propagation of the quantum string modes, it is easy to see that the propagation of the tachyon field in this background remains the same as in empty space: the dilaton dependence in

$$
-\frac{1}{2} D^{2} T+\partial^{\mu} \phi \partial_{\mu} T-\frac{2}{\alpha^{\prime}} T=-\frac{1}{2} \partial_{i}^{2} T-\partial_{u} \partial_{v} T+\partial_{u} \phi \partial_{v} T-\frac{2}{\alpha^{\prime}} T
$$

can be completely eliminated by $T \rightarrow T^{\prime}=\mathrm{e}^{\phi(u)} T$. Similar conclusion holds for the propagators of other modes except the graviton one (the graviton propagator is changed from the flat one by $O\left(\dot{b}_{i j}^{2}\right)$-dependent terms). The string interaction vertices depend of course on $\phi(u)$ (and some also on $\dot{b}_{i j}$ ) so that the scattering of strings on this background should be quite non-trivial.

Another special case is $\phi=$ const. Then eq.(3) is satisfied due to the cancellation between the Ricci tensor and the antisymmetric tensor field strength contributions as for parallelisable spaces or WZW models [31]. In fact, a particular model of this type (with $D=2$ ) was recently interpreted [32] as a WZW theory based on a non-semisimple group (a central extension of the 2-dimensional Euclidean group). The corresponding action can be put into the form

$I(u, v, x)=\frac{1}{\pi \alpha^{\prime}} \int d^{2} z\left(-2 \partial u \bar{\partial} v+\partial x_{1} \bar{\partial} x_{1}+\partial x_{2} \bar{\partial} x_{2}+2 \cos u \partial x_{1} \bar{\partial} x_{2}\right)+\frac{1}{4 \pi} \int d^{2} z \sqrt{\gamma} R^{(2)} \phi_{0}$.

The structure of this action is very simple: if one formally replaces $-2 \partial u \bar{\partial} v$ - term by $\partial u \bar{\partial} u$ then (4) becomes the action of the $S U(2)$ WZW model. One can also make the 
complex rotation $u^{\prime}=i u, v^{\prime}=-i v$ replacing $\cos u$ by $\cosh u$ and thus going from $S U(2)$ to $S L(2, R)$. This observation implies that the $O(2,2)$ duality transformations of (4) with respect to $x_{1}, x_{2}$ look the same (with $\partial u \bar{\partial} u \rightarrow-2 \partial u \bar{\partial} v$ ) as the duality rotations of the $S U(2)$ WZW model (discussed in [19] 33] [34] 20] 35]). For example, performing the duality transformation in the $\left(x_{1}+x_{2}\right)$-direction we get

$I^{\prime}=\frac{1}{\pi \alpha^{\prime}} \int d^{2} z\left[-2 \partial u \bar{\partial} v+\operatorname{tg}^{2}\left(\frac{u}{2}\right) \partial y_{1} \bar{\partial} y_{1}+\partial y_{2} \bar{\partial} y_{2}\right]+\frac{1}{4 \pi} \int d^{2} z \sqrt{\gamma} R^{(2)}\left[\phi_{0}-\frac{1}{2} \ln (1+\cos u)\right]$

Another example is a partucular $O(2,2)$ duality transformation [20] that leads from from the 'charged black hole' $([S L(2, R) \times R] / R$ gauged WZW $)$ model of [26]

$$
\begin{gathered}
I^{\prime \prime}=\frac{1}{\pi \alpha^{\prime}} \int d^{2} z\left\{-2 \partial u \bar{\partial} v+\frac{(\lambda-1) \sin ^{2} \frac{u}{2}}{\cos ^{2} \frac{u}{2}-\lambda} \partial y_{1} \bar{\partial} y_{1}+\frac{\lambda \cos ^{2} \frac{u}{2}}{\cos ^{2} \frac{u}{2}-\lambda} \partial y_{2} \bar{\partial} y_{2}\right. \\
\left.-\frac{\lambda \sin ^{2} \frac{u}{2}}{\cos ^{2} \frac{u}{2}-\lambda}\left(\partial y_{1} \bar{\partial} y_{2}-\partial y_{2} \bar{\partial} y_{1}\right)\right\}+\frac{1}{4 \pi} \int d^{2} z \sqrt{\gamma} R^{(2)}\left[\phi_{0}-\frac{1}{2} \ln (1-2 \lambda+\cos u)\right]
\end{gathered}
$$

where $\lambda$ is a free parameter. The models $\left(4^{\prime}\right),\left(4^{\prime \prime}\right)$ belong to the same class (1). Since the standard leading-order duality rotations we have applied preserve the one-loop string equations (i.e. preserve (3), see below) the backgrounds in $\left(4^{\prime}\right),\left(4^{\prime \prime}\right)$ also represent exact string solutions.

3. Let us now discuss systematically the action of the $O(D, D \mid R)$ duality on the above class (1) of exact string solutions. First, let us demonstrate explicitly that eq.(3) is invariant under $O(D, D \mid R)$ duality. The argument is essentially the same as in the timedependent case of [17] (but even simpler). 0 Introducing the basic $2 D \times 2 D$ matrix $M(u)$

5 It is possible to check the invariance of the string effective action as in 17] by introducing the Lagrange-multiplier field $G^{u u}$ (not transforming under the duality) the variation over which gives (3). Then the string effective action takes the form:

$$
\int d u \mathrm{e}^{-2 \varphi}\left\{-\frac{2}{3}(D-24)+\alpha^{\prime} G^{u u}\left[\frac{1}{8} \operatorname{Tr}(\dot{M} \eta \dot{M} \eta)+2 \ddot{\varphi}\right]\right\}
$$


[12] built out of $g_{i j}(u)$ and $b_{i j}(u)$, the constant matrix $\eta$ and the duality-invariant dilaton $\varphi(u)$ [16] 15

$$
\begin{aligned}
M & =\left(\begin{array}{cc}
g^{-1} & -g^{-1} b \\
b g^{-1} & g-b g^{-1} b
\end{array}\right), \quad \eta=\left(\begin{array}{cc}
0 & I \\
I & 0
\end{array}\right), \\
\varphi & =\phi-\frac{1}{4} \ln \operatorname{det} g, \quad \mathrm{e}^{-2 \varphi}=\mathrm{e}^{-2 \phi} \sqrt{g},
\end{aligned}
$$

one can represent (3) in the form

$$
\frac{1}{8} \operatorname{Tr}(\dot{M} \eta \dot{M} \eta)+2 \ddot{\varphi}=0
$$

which is manifestly invariant under the $O(D, D \mid R)$ transformations (with a constant parameter matrix $\Lambda$ )

$$
M^{\prime}=\Lambda M \Lambda^{T}, \quad \varphi^{\prime}=\varphi, \quad \Lambda^{T} \eta \Lambda=\eta .
$$

The two terms in (7) are particular invariants of the duality group. The class of backgrounds corresponding to (1) splits into orbits under the action (8) of $O(D, D \mid R)$. For example, let us consider the orbit that contains the flat background $g_{i j}=\delta_{i j}$. It is clear that (some) duality transformations will rotate this flat background into curved ones. This is similar to the well-known example of the duality transformation acting on flat space in flat coordinates [19] [17]: $d s^{2}=d r^{2}+r^{2} d \theta^{2} \rightarrow d s^{2}=d r^{2}+r^{-2} d \theta^{2}$. However, in our case both the original and the duality transformed backgrounds are known explicitly since they are not modified by $\alpha^{\prime}$-corrections. For example, consider the following $D+2=4$ background

$$
g_{i j}=\left(\begin{array}{cc}
1 & f \\
f & 1+f^{2}
\end{array}\right), \quad b_{i j}=0, \quad f=f(u), \quad \phi=\phi(u),
$$

where $f$ and $\phi$ are related by (3), i.e. $R_{u u}=-\frac{1}{2} \dot{f}^{2}=-2 \ddot{\phi}$. After the duality rotation in the $x_{1}$-direction we get

$$
g_{i j}^{\prime}=\delta_{i j}, \quad b_{i j}^{\prime}=-f \epsilon_{i j}, \quad \phi^{\prime}=\phi,
$$

i.e. we have transformed a curved $\left(R_{i u j u} \neq 0\right)$ four-dimensional background into the one with a flat metric (but non-trivial antisymmetric tensor and dilaton). 
If we assume that the spatial directions $x^{i}$ form a torus then the duality subgroup $O(D, D \mid z)$ will generate backgrounds that all correspond to the same conformal field theory [19] 20]. In particular, (9) and (10) thus give different space-time representations of the same exact string solution. Another example is provided by $(4)$ and $\left(4^{\prime}\right)$ and $\left(4^{\prime \prime}\right)$.

4. In addition to the $x^{i}$-isometries, the action (1) is also invariant under the constant shifts of $v$ corresponding to the null Killing vector. This is a novel situation absent in the case of the 'cosmological' backgrounds of [17]. One is thus tempted to consider more general $O(D+1, D+1)$ rotations of the models (1). Performing the duality 'inversion' in the null direction leads to a singularity. One could hope that inclusion of other non-null isometry directions may 'regularize' this singularity. Formally, the string equations should still be invariant under such duality rotations. In general, $O(D+1, D+1)$ transformations move us out of our class of exact null backgrounds (1). However, a subset of transformations which contains $O(D, D)$ transformations combined with 'triangular' $G L(D+1, R)$ coordinate transformations $v^{\prime}=a v+c_{i} x^{i}, x^{\prime}=d_{j}^{i} x^{j}$ still preserves the class (1).

Let us now show that first making a coordinate transformation and then such an $O(3,3)$ duality rotation including the $G L(3, R)$ shift of $v$ it is possible to transform the model (4) into the trivial one (with flat $g_{i j}$ and constant $b_{i j}, \phi$ ). Changing the coordinates $\left(u, v, x_{1}, x_{2}\right) \rightarrow\left(u, v^{\prime}, r, \theta\right)$

$$
r \cos \theta=x_{1}+x_{2} \cos u, \quad r \sin \theta=x_{2} \sin u, \quad v^{\prime}=v-\frac{1}{2} x_{1} x_{2} \sin u,
$$

one can put (4) into the form

$$
I(u, v, r, \theta)=\frac{1}{\pi \alpha^{\prime}} \int d^{2} z\left(-2 \partial u \bar{\partial} v^{\prime}+\partial r \bar{\partial} r+r^{2} \partial \theta \bar{\partial} \theta-2 r^{2} \partial u \bar{\partial} \theta\right)+\frac{1}{4 \pi} \int d^{2} z \sqrt{\gamma} R^{(2)} \phi_{0} .
$$

The simple duality with respect to $\theta$ transforms (11) into

$$
\begin{gathered}
I^{\prime}\left(u, v, r \theta^{\prime}\right)=\frac{1}{\pi \alpha^{\prime}} \int d^{2} z\left(-2 \partial u \bar{\partial} v^{\prime}+\partial r \bar{\partial} r+r^{-2} \partial \theta^{\prime} \bar{\partial} \theta^{\prime}-\frac{1}{2} \partial u \bar{\partial} \theta^{\prime}-\frac{1}{2} \bar{\partial} u \partial \theta^{\prime}\right) \\
+\frac{1}{4 \pi} \int d^{2} z \sqrt{\gamma} R^{(2)}\left(\phi_{0}-\frac{1}{2} \ln r\right) .
\end{gathered}
$$


If we now shift $v^{\prime} \rightarrow v+\frac{1}{2} \theta^{\prime}$ and perform the duality with respect to $\theta^{\prime}$ we get precisely the flat sigma model with constant antisymmetric tensor and dilaton. One is thus able to relate the model (4) to the flat one by combining a peculiar coordinate transformation with two $O(2,2)$ rotations and a linear shift of $v$ (as noted above, the latter can be considered as an element of $O(3,3)$ rotation $)$. 6

In conclusion, let us emphasize the important role played by the antisymmetric tensor background in the models (1). Since the duality intertwines the metric and the antisymmetric tensor, in some cases the antisymmetric tensor background may play the role (from a string point of view) of a curved metric background. We hope that further study of the class of backgrounds (1) may help to elucidate the role of duality as a (part of) generalized 'coordinate invariance' in string theory.

6 While typing this paper we have learned about a recent preprint [36] in which a possibility to transform the background of 32] to flat space by a pure $O(2,2)$ duality rotation is mentioned. We were also informed that a duality rotation of a gauged version of the model of [32] also leading to a flat space was discussed in [37]. 


\section{References}

[1] H.W. Brinkmann, Math. Ann. 94(1925)119.

[2] R. Guven, Phys. Lett. B191(1987)275.

[3] D. Amati and C. Klimčík, Phys. Lett. B219(1989)443.

[4] G. Horowitz and A.R. Steif, Phys.Rev.Lett. 64(1990)260 ; Phys.Rev. D42(1990)1950.

[5] G. Horowitz, in: Proceedings of Strings '90, College Station, Texas, March 1990 (World Scientific,1991).

[6] R.E. Rudd, Nucl. Phys. B352(1991)489 .

[7] C.Duval, G.W. Gibbons and P.A. Horváthy, Phys. Rev. D43(1991)3907 ; C.Duval, G.W. Gibbons, P.A. Horváthy and M.J. Perry, unpublished (1991); C. Duval, Z. Horvath and P.A. Horváthy, Phys. Lett. B313(1993)10; Marseille preprints (1993).

[8] E.A. Bergshoeff, R. Kallosh and T. Ortin, Phys. Rev. D47(1993) 5444.

[9] A.A. Tseytlin, Phys. Lett. B288(1992)279; Phys. Rev. D47(1993)3421.

[10] A.A. Tseytlin, Nucl. Phys. B390(1993)153.

[11] G. Horowitz and A.A. Steif, Phys. Lett. B250(1990)49; E. Smith and J. Polchinski, Phys. Lett. B263(1991)59; J. Horne, G. Horowitz and A. Steif, Phys. Rev. Lett. 68(1992)568; G. Horowitz, in; Proc. of the 1992 Trieste Spring School on String theory and Quantum Gravity, preprint UCSBTH-92-32, hep-th/9210119; G. Horowitz and D.L. Welch, Phys. Rev. Lett. 71(1993)328.

[12] A. Giveon, E. Rabinovici and G. Veneziano, Nucl. Phys. B322(1989)167; A. Shapere and F. Wilczek, Nucl. Phys. B320(1989)669.

[13] A. Giveon, N. Malkin and E. Rabinovici, Phys. Lett. B238(1990)57.

[14] T.H. Buscher, Phys. Lett. B194(1987)59 ; Phys. Lett. B201(1988)466.

[15] A.A. Tseytlin, Mod. Phys. Lett. A6(1991)1721.

[16] G. Veneziano, Phys. Lett. B265(1991)287.

[17] K.M. Meissner and G. Veneziano, Phys. Lett. B267(1991)33; M. Gasperini, J. Maharana and G. Veneziano, Phys. Lett. B272(1991)277; Phys. Lett. B296(1992)51.

[18] A. Sen, Phys. Lett. B271(1991)295; Phys. Lett. B274(1991)34.

[19] M. Roček and E. Verlinde, Nucl. Phys. B373(1992)630.

[20] A. Giveon and M. Roček, Nucl. Phys. B380(1992)128.

[21] E. Alvarez, L. Alvarez-Gaumé, J. Barbón and Y. Lozano, preprint CERN-TH.6991/93.

[22] A.A. Tseytlin, Nucl. Phys. B350(1991)395.

[23] J. Panvel, Phys. Lett. B284(1992)50.

[24] A. Giveon, Mod. Phys. Lett. A6(1991)2843; R. Dijkgraaf, H. Verlinde and E. Verlinde, Nucl. Phys. B371(1992)269; I. Bars, preprint USC-91-HEP-B3; E. Kiritsis, Mod. Phys. Lett. A6(1991)2871.

[25] A.A. Tseytlin, preprint CERN-TH.6970/93; hep-th/9308042.

[26] J.H. Horne and G.T. Horowitz, Nucl. Phys. B368(1992)444. 
[27] A. Giveon and E. Kiritsis, preprint CERN-TH.6816/93; hep-th/9303016.

[28] K. Sfetsos and A.A. Tseytlin, preprint CERN-TH.6969/93, hep-th/9310159.

[29] K.S. Narain, M.H. Sarmadi and E. Witten, Nucl. Phys. B279(1987)369.

[30] R. Myers, Phys. Lett. B199(1987)371; I. Antoniadis, C. Bachas, J. Ellis, D. Nanopoulos, Phys. Lett. B211(1988)393; Nucl. Phys. B328(1989)115.

[31] E. Witten, Commun. Math. Phys. 92(1984)455 ; E. Braaten, T.L. Curtright and C.K. Zachos, Nucl. Phys. B260(1985)630.

[32] C. Nappi and E. Witten, Princeton preprint IAS-HEP-93/61; hep-th/9310112.

[33] A. Kumar, Phys. Lett. B293(1992)49; D. Gershon, preprint TAUP-2005-92.

[34] S. Hussan and A. Sen, Nucl. Phys. B405(1993)143.

[35] E. Kiritsis, Nucl. Phys. B405(1993)109.

[36] K. Kounnas and E. Kiritsis, preprint CERN-TH.7059/93; hep-th/9310202.

[37] K. Sfetsos, to appear. 